\title{
RF Exposure Analysis for Multiple Wi-Fi Devices In Enclosed Environment
}

\author{
Shian U. Hwu ${ }^{1}$, Bryan A. Rhodes ${ }^{2}$, \\ B. Kanishka deSilva ${ }^{2}$ \\ ${ }^{1}$ Barrios Technology, ${ }^{2}$ Jacobs Technology \\ Houston, Texas, USA \\ Shian.u.hwu@nasa.gov
}

\author{
Catherine C. Sham, James R. Keiser \\ NASA/JSC/EV \\ NASA Johnson Space Center \\ Houston, Texas, USA \\ Catherine.C.Sham@nasa.gov
}

\begin{abstract}
Wi-Fi devices operated inside a metallic enclosure have been investigation in the recent years. A motivation for this study is to investigate wave propagation inside an enclosed environment such as elevator, car, aircraft, and spacecraft. There are performances and safety concerns when RF transmitters are used inside a metallic enclosed environment. In this paper, the field distributions inside a closed metallic room were investigated with multiple portable Wi-Fi devices. Computer simulations were performed using rigorous computational electromagnetics (CEM). The method of moments (MoM) was used to model the mutual coupling among antennas. The geometrical theory of diffraction (GTD) was applied for the multiple reflections off the floor and walls. The prediction of the field distribution inside such an environment is useful for the planning and deployment of a wireless radio and sensor system. Factors that affect the field strengths and distributions of radio waves in confined space were analyzed. The results could be used to evaluate the RF exposure safety in confined environment. By comparing the field distributions for various scenarios, it was observed that the Wi-Fi device count, spacing between the devices and relative locations in the room are important factors in the deployment of these devices. The RF Keep Out Zone (KOZ), where the electric field strengths exceed the permissible RF exposure limit, could be used to assess the RF human exposure compliance. This study shows, it's possible to maximize or minimize field intensity in specific area by arranging the Wi-Fi devices as a function of the relative location and spacing.
\end{abstract}

Keywords- RF exposure; metallic enclosure; Wi-Fi device; WLAN; Acess Point; Laptop; Tablet; Computational Electromagnetics, propagation.

\section{INTRODUCTION}

The use of Wi-Fi devices such as notebook computers, tablets, PDAs, and smart phones has seen rapid growth in recent years. At the same time, the concern of the RadioFrequency (RF) radiation effects on human health due to the Wi-Fi devices by the consumer has also increased. RF safety is an important issue for the Wi-Fi device manufacturers, consumers, and the government regulators.

The RF exposure standards on the permissible radiation levels of consumer Wi-Fi devices are regulated by the International Commission on Non-Ionizing Radiation Protection (ICNIRP) guidelines and the IEEE C95.1 standard [4]. The Wi-Fi devices must be designed such that the radiated field strength from the Wi-Fi transmitter is within the permissible exposure limits. These permissible exposure limit standards are based on the thermal effects of the RF energy in the human body.

Wi-Fi devices operated inside a metallic enclosure have been investigation in the recent years [2-4]. A motivation for this study is to analyzed wave propagation inside an enclosed environment such as elevator, car, aircraft, or spacecraft. The health risk could be higher for the RF transmitters, such as Wi-Fi devices, used in a confined environment. This concern is based on the fact that the RF energy will not have any escape route and all the energy emitted by the RF emitters will be absorbed by the occupants. .

In an enclosed environment, the transmit power of some advanced smart Wi-Fi devices could automatically increase to compensate for a weak received signal. Because of the wave reflections, electric field in enclosed environment could be very complicated with constructive and destructive interferences. The constructive interference could intensify with multiple reflections.

There has been concerned that a metallic enclosure could potentially act like an imperfect resonant cavity, leading to RF hot spots where the electromagnetic fields are enhanced $[5,6]$. This could increase the severity of the RF exposure for the Wi-Fi device users in such an environment.

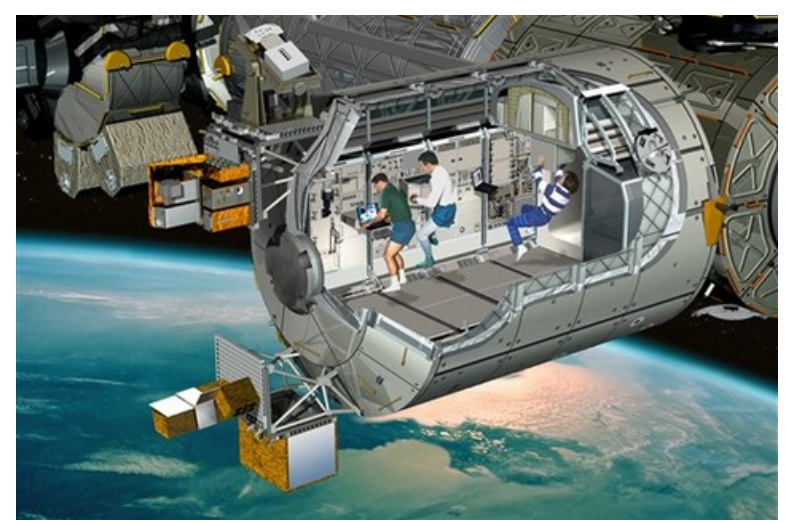

Figure 1. International Space Station consists of many enclosed metallic modules.

The distribution of the electric field from the multiple $\mathrm{Wi}-\mathrm{Fi}$ transmitters is calculated using rigorous computational electromagnetics (CEM). The method of moments (MoM) was used to model the mutual coupling among antennas [7]. The geometrical theory of diffraction (GTD) was applied for the multiple reflections off the floor and walls [8]. The RF Keep Out Zone (KOZ), where the calculated electric field 
strength exceeds the permissible RF exposure limit, could be determined to assess the RF human exposure compliance.

\section{SimUlation ResUlts}

The enclosed indoor environment is modeled as a metallic box sized $2 \mathrm{~m}$ by $2 \mathrm{~m}$ by $2 \mathrm{~m}$. A deterministic ray tracing algorithm, the geometrical theory of diffraction is used for the field interactions including multiple reflections from the floor and walls. The multiple Wi-Fi devices were modeled as half-wavelength dipole antennas placed at various separation distances from each other. The transmit power is assumed to be $0.25 \mathrm{~W}$ or $24 \mathrm{dBm}$ which is typical for consumer Wi-Fi devices. The industrial, scientific and medical (ISM) radio frequency band at $2.4 \mathrm{GHz}$ is assumed. The antennas were modeled using the rigorous method of moments. The antenna mutual coupling effects were taken into accounts.

\section{A. Confined Space}

Figure 2 shows the field intensity for a Wi-Fi antenna (a) placed in an opened space (on the left) and (b) placed in a confined metallic (perfectly reflective) room sized of $2 \mathrm{~m}$ by $2 \mathrm{~m}$ by $2 \mathrm{~m}$ (on the right). Figure 3 shows the field intensity for two Wi-Fi antennas (a) placed in an opened space (on the left) and (b) placed in a confined room sized of $2 \mathrm{~m}$ by $2 \mathrm{~m}$ by $2 \mathrm{~m}$ (on the right). The spacing between the Wi-Fi antennas is 2 wavelengths.

Figure 4 shows the differences of the electric fields between in the confined space and in free space. It can be observed that the field strengths could increase significantly for $\mathrm{Wi}-\mathrm{Fi}$ device operated in a confined room than in an opened space. The total signal increases due to constructive interference where the direct signal and the reflected signals are in phase. A smaller room could have more pronounce effect than a larger room.

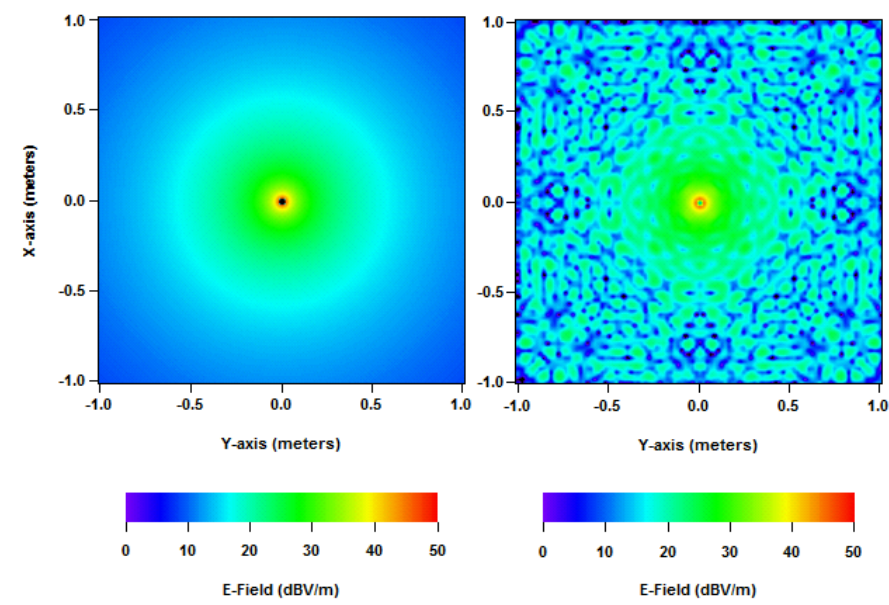

Figure 2. Electric field distributions for one Wi-Fi device in free space (Left) and in a confined space (Right).
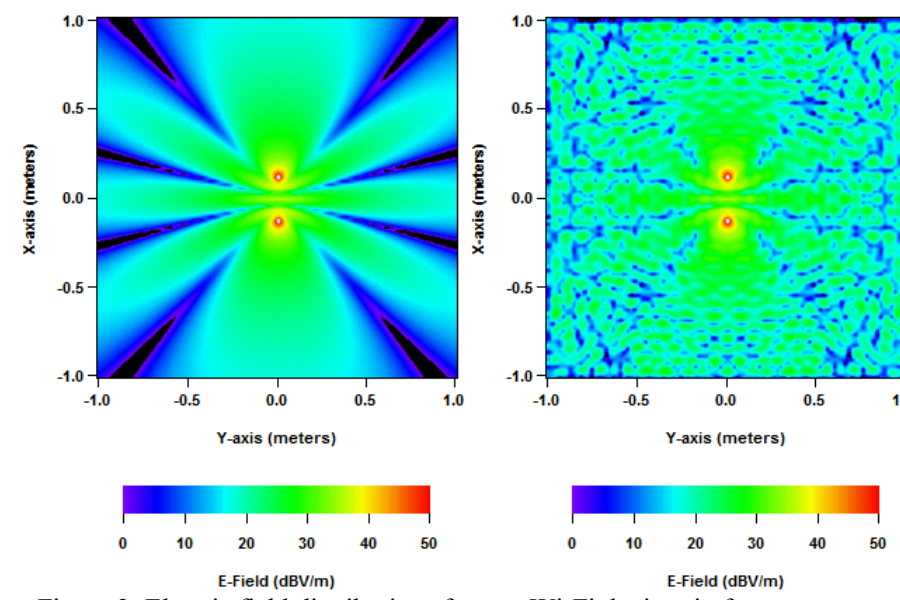

Figure 3. Electric field distributions for two Wi-Fi devices in free space (Left) and in a confined environment (Right).

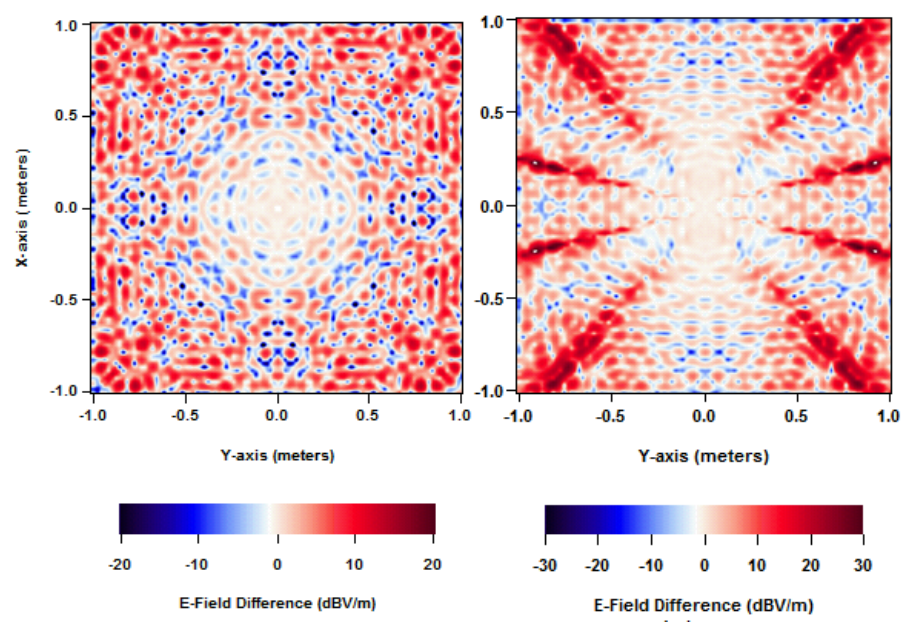

Figure 4. Differences of electric fields between in a confined space and in free space for one (Left) and two (Right) Wi-Fi devices.

\section{B. Spacing Between Wi-Fi Devices}

Figure 5 shows the field intensity for two Wi-Fi antennas placed $1 \lambda$ (Left) and $1.5 \lambda$ (Right) apart in a closed room sized, $2 \mathrm{~m}$ by $2 \mathrm{~m}$ by $2 \mathrm{~m}$. Figure 6 shows the field intensity for three Wi-Fi antennas placed $1 \lambda$ (Left) and $1.5 \lambda$ (Right) apart. It can be observed that the field distributions are very different with different spacing among the Wi-Fi devices. The mutual coupling effects are determined by the antenna spacing. The RF energy radiation patterns (i.e., stronger in some directions) can be changed by varying the antenna spacing. Thus, the Wi-Fi device spacing has a large influence on the field distributions.

\section{Number of Wi-Fi Devices}

Figure 7 shows the electric field distributions for one (Left) and two (Right) Wi-Fi devices placed near the upper right corner of a closed room. It can be observed that the field strength increases significantly by adding multiple $\mathrm{Wi}$ Fi devices. Figure 8 shows the difference in electric field with two (Left) and four Wi-Fi devices (Right). At some locations (blue regions), the fields are reduced. The reason is destructive interferences among the different electric field 
components with significant phase difference. The number of the devices and the locations were changed for the four device case in Figure 8.
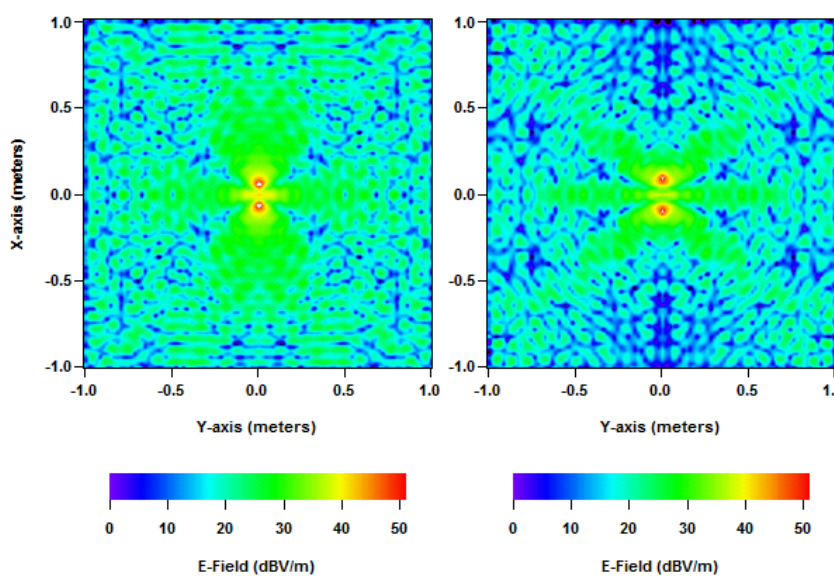

Figure 5. Electric field distributions for two Wi-Fi devices with $1 \lambda$ (Left)

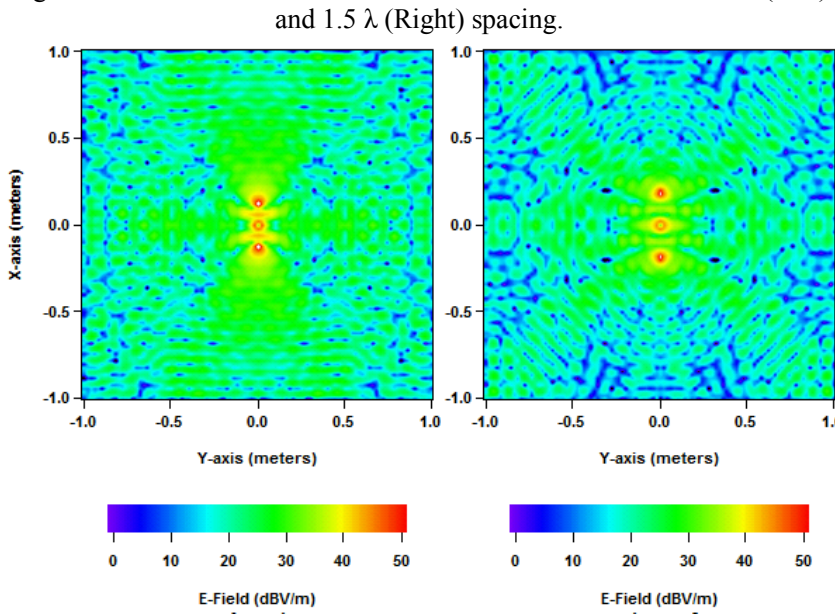

Figure 6. Electric field distributions for three Wi-Fi devices with $1 \lambda$ (Left)

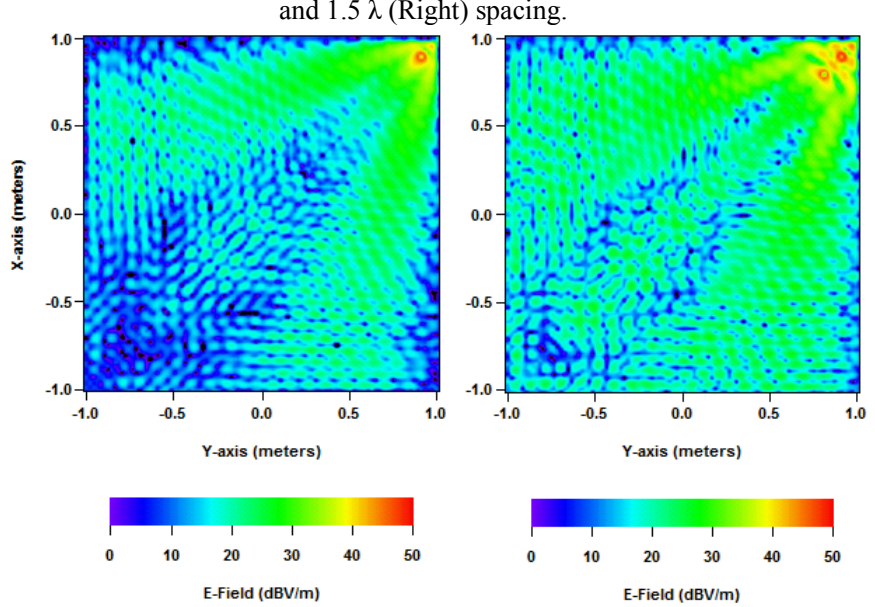

Figure 7. Electric field distributions for one (Left) and two (Right) Wi-Fi devices near the upper right corner.
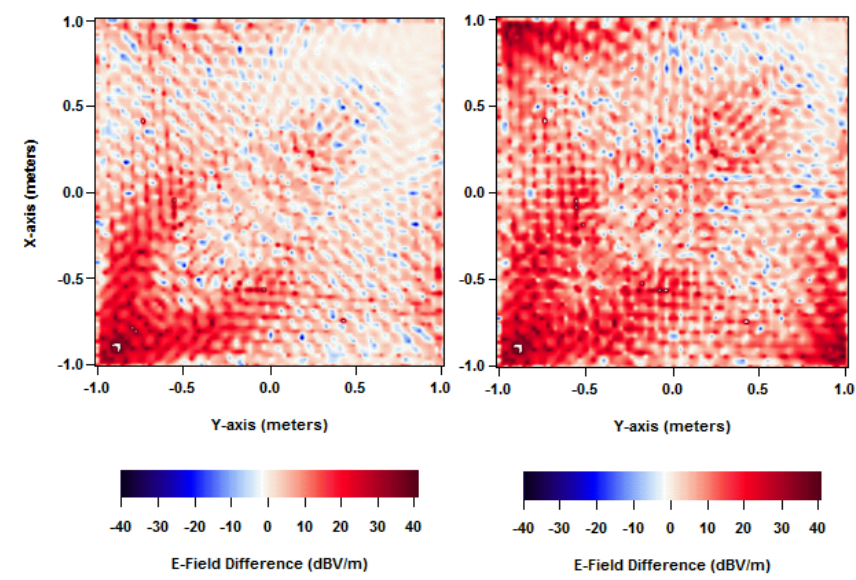

Figure 8. Electric field increases with multiple Wi-Fi devices as shown for two (Left) and four devices (Right).

\section{Locations of Wi-Fi Devices}

The Wi-Fi device locations can have a large influence on the field distributions. As shown in Figures 9 and 10, four Wi-Fi devices are placed near the center and at the corners of the closed room.

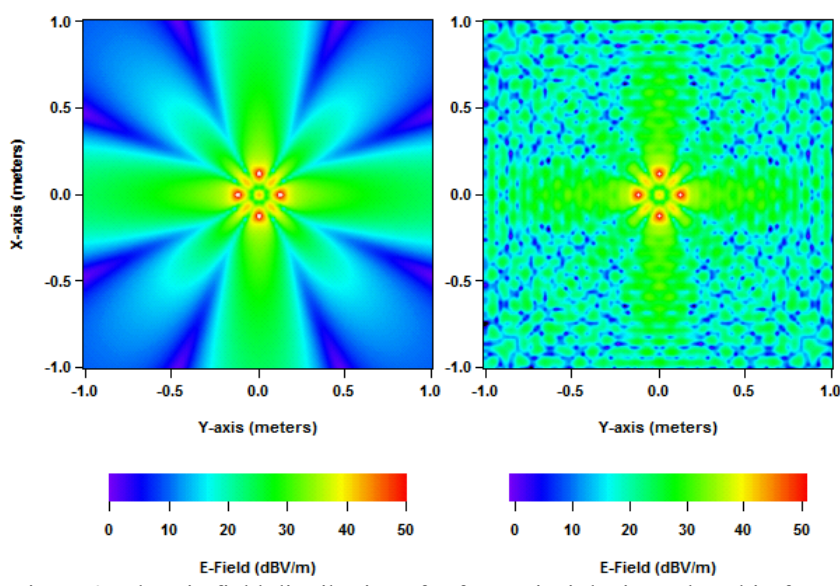

Figure 9. Electric field distributions for four Wi-Fi devices placed in free space (Left) and placed in a confined environment (Right) near the center.
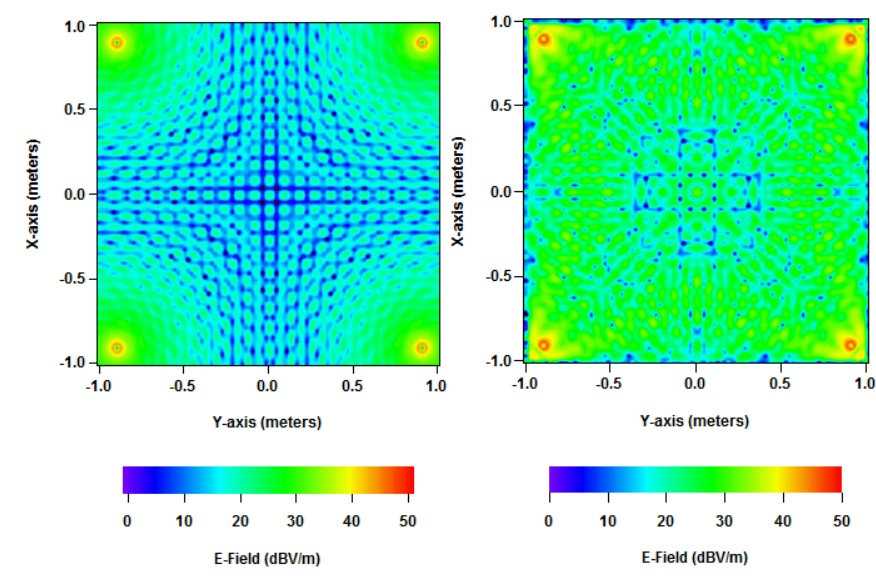

Figure 10. Electric field distributions for four Wi-Fi devices placed in free space (Left) and placed in a confined environment (Right) near the corners. 


\section{CONCLUSION}

A numerical study on RF radiation of multiple Wi-Fi devices inside a confined space is presented in this paper. The effects of number of Wi-Fi devices and their positions inside the room on the electric field intensities and distributions were investigated. Results have shown that the electric field could be significantly affected by the location of placement and the number of the Wi-Fi devices. The electric fields are enhanced due to mutual coupling among the Wi-Fi devices and the multiple reflections from the ground and walls. No cavity or resonance effects were observed for the $2.4 \mathrm{GHz}$ ISM band devices in the room modeled and the number of devices and locations investigated. The resonance effects likely require smaller enclosed space with lower frequencies. As shown in this study, it's possible to maximize or minimize field intensity in specific area by arranging the Wi-Fi devices in a planned placement and spacing. Since the radiation is maximal in the plane perpendicular to the dipole antenna and minimum in the direction along the antenna axis. The antenna coupling effects could be reduced if the antennas are placed not in the same plane.

\section{REFERENCES}

[1] Simba, A.Y.; Watanabe, S.; Hikage, T.; Nojima, T.; , "A review of mobile phone usage in enclosed areas and RF safety guideline," AFRICON, 2009. AFRICON '09. , vol., no., pp.1-6, 23-25 Sept. 2009

[2] Sai-Wing Leung; Yinliang Diao; Kwok-Hung Chan; Yun-Ming Siu; Yongle Wu; , "Specific Absorption Rate Evaluation for Passengers
Using Wireless Communication Devices Inside Vehicles With Different Handedness, Passenger Counts, and Seating Locations," Biomedical Engineering, IEEE Transactions on , vol.59, no.10, pp.2905-2912, Oct. 2012

[3] Tang, C.K.; Chan, K.H.; Fung, L.C.; Leung, S.W.; , "Antenna performance of mobile phone and corresponding human exposure inside fully and partially enclosed metallic elevator," Electromagnetic Compatibility, 2008. EMC 2008. IEEE International Symposium on, vol., no., pp.1-5, 18-22 Aug. 2008

[4] IEEE Standard for Safety Levels With Respect to Human Exposure to Radio Frequency Electromagnetic Fields IEEE, $3 \mathrm{kHz}$ to $300 \mathrm{GHz}$ IEEE Std C95.1-2005, 2005.

[5] Bamba, A.; Joseph, W.; Andersen, J.B.; Tanghe, E.; Vermeeren, G.; Plets, D.; Nielsen, J.O.; Martens, L.; , "Experimental Assessment of Specific Absorption Rate Using Room Electromagnetics," Electromagnetic Compatibility, IEEE Transactions on , vol.54, no.4, pp.747-757, Aug. 2012

[6] Robert G. Olsen; Richard A. Tell; , "Evaluation of Protective Hoods in Strong RF Electromagnetic Fields," Power Delivery, IEEE Transactions on, vol.22, no.1, pp.340-346, Jan. 2007

[7] Hwu, S.U.; Wilton, D.R.; Rao, S.M., "Electromagnetic scattering and radiation by arbitrary conducting wire/surface configurations," Antennas and Propagation Society International Symposium, 1988. AP-S. Digest, Syracuse, NY, 6-10 June 1988 Page(s):890 - 893 vol.2

[8] Marhefka, R.J., and Silvestro, J.W., "Near zone - basic scattering code user's manual with space station applications," NASA CR181944, Dec. 1989.

[9] Hwu, S.U.; Yin-Chung Loh ; Sham, C.C.; Kroll, Q.D. "Space Shuttle and Space Station radio frequency (RF) exposure analysis," IEEE Digital Avionics Systems Conference (DASC), 30 Oct.-3 Nov. 2005. 